Check for updates

Cite this: RSC Adv., 2019, 9, 14708

\title{
In vitro and in vivo evaluation of a novel mitomycin nanomicelle delivery system $\dagger$
}

\author{
Hongmei Yang, (D) a Miao Wang, ${ }^{a}$ Yihe Huang, ${ }^{\text {bc }}$ Qiaoyu Qiao, ${ }^{a}$ Chunjie Zhao ${ }^{a}$ \\ and Min Zhao (D) *a
}

Mitomycin C (MMC), naturally synthesized by Streptomyces caespitosus, is a potent antineoplastic antibiotic for the treatment of various solid tumors. However, the defects of conventional MMC injections have greatly limited its clinical application due to its toxic side effects and non-specific interactions. To solve this problem, the $\mathrm{PEG}_{2 \mathrm{k}}$-Fmoc-lbuprofen (PEG-Flbu) micellar nanocarrier was synthesized and the MMCloaded micelles (PEG-Flbu/MMC) were prepared by thin film hydration method and characterized. Ibuprofen was used as a hydrophobic domain of PEG-Flbu nanocarrier, and we expect it to synergize with codelivered MMC in the overall antitumor activity. The in vitro release of PEG-Flbu/MMC was examined by dialysis method using MMC injection as a control. Our data suggested that PEG-Flbu/MMC micelles presented appropriate particle size, low CMC value, good stability, high drug loading efficiency and sustained release properties. In vitro cytotoxicity studies with several tumor cell lines showed that the carrier was effective in mediating intracellular delivery of MMC to tumor cells. In vivo pharmacokinetics, tissue distribution and therapeutic study proved that PEG-FIbu/MMC micelles prolonged blood circulation, significantly improved the tumor accumulation and therapeutic efficacy, and reduced undesirable side effect on normal tissues compared to MMC injection. In general, PEG-Flbu/MMC micelles represented an effective strategy to improve the performance for the delivery of $M M C$ and safety of medication.

Received 9th April 2019
Accepted 7th May 2019

DOI: $10.1039 / \mathrm{c} 9 \mathrm{ra02660f}$

rsc.li/rsc-advances reduce the adverse effect of MMC injection which has been used clinically by utilizing nano-delivery systems (namely: including polymeric micelles, liposomes, dendrimers and nanoparticles)..$^{5-12}$ It is well known that macromolecular and nano carriers tend to preferentially accumulate within tumors owing to the enhanced penetration and retention (EPR) effect, ${ }^{13,14}$ which offers a momentous opportunity for selective delivery of antitumor drugs. ${ }^{5}$

Polymeric micelles are recognized as a promising delivery system for their easy preparation, small size, sustained release and extended blood circulation time. ${ }^{15}$ A variety of drugs conjugated of copolymers by non-covalent interactions were solubilized and then escaped uptake by the reticuloendothelial system (RES), thereby increased drug loading and stability. ${ }^{16,17}$ We previously developed $\mathrm{PEG}_{2 \mathrm{k}}$-Fmoc-Ibuprofen (PEG-FIbu) micellar nanocarrier that were highly efficient in formulating paclitaxel (PTX) and obtained synergistic efficacy compared to Taxol which is a clinically used PTX formulation in vivo. ${ }^{18}$ PEGFIbu composed of PEG hydrophilic chains acted as outer shell, a Fmoc motif, and an inner core of hydrophobic segment based on ibuprofen. PEG is a linear polymer with good water solubility, biocompatibility, non-toxic, non-immunogenic, nonteratogenic and non-antigenic. Fmoc incorporated into nanocarriers as interfacial drug-interactive motifs not merely improved the carrier/drug interaction, but also promoted the interaction among the carrier molecules themselves, which has
${ }^{a}$ School of Pharmacy, Shenyang Pharmaceutical University, No. 103, Wenhua Road, Shenyang 110016, China. E-mail: lab433@163.com; Fax: +86-24-23986299; Tel: +86-24-43520597

${ }^{b}$ College of Chemistry, Liaoning University, Shenyang 110036, China ${ }^{c}$ Shenyang Medical College, Shenyang 110034, China

$\dagger$ Electronic supplementary information (ESI) available. See DOI: 10.1039/c9ra02660f 

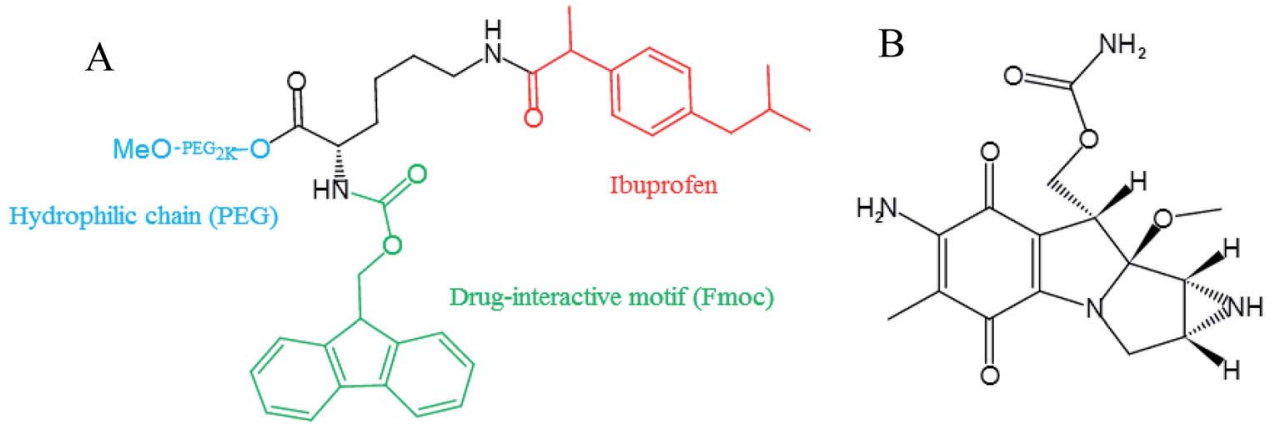

Fig. 1 Chemical structures formula of PEG-Flbu (A) and MMC (B).

recently been discovered as an effective strategy to enhance carrier-drug compatibility of micellar system. ${ }^{19-22}$ There is a possibility for its cleavage and a certain degree of cytotoxicity according to Hegmans's work. ${ }^{23}$ It shows selective release of cytotoxic drug through hydrolytic cleavage of carbamates in tumor tissue. Fmoc is a protecting group formed by carbamate structure and fluorene. It can be hydrolyzed by hydrolase, and the possible toxicity mainly comes from fluorenyl. At present, fluorenyl group is rarely found in drug molecules. Because of its poor water solubility, fluorenyl group is generally not used in small molecular drugs. However, the metabolism of fluorenyl is generally believed to be oxidized by P450 to form gluconate or sulfate, which can be discharged. ${ }^{24} \mathrm{~A}$ large number of epidemiological studies and in vivo and in vitro experiments have shown that ibuprofen can inhibit the proliferation of many malignant tumors. Long-term and frequent use of ibuprofen can reduce the incidence of malignant tumors. ${ }^{25,26}$ Different from majority of the existing drug carriers which devoid of therapeutic activity, PEG-FIbu itself presents anticancer efficacy that is attributed to the effect of ibuprofen on suppressing tumor cell proliferation. ${ }^{18}$

This is the first investigation in which the antitumor antibiotic is exploited with the PEG-FIbu nanocarrier to construct the dual functional nanomicelles delivery system for enhanced targeted delivery of MMC to obtain increased therapeutic efficacy and reduced toxicity compared to the conventional MMC injection. The PEG-FIbu micelles carrier was synthesized and MMC-loaded PEG-FIbu micelles (PEG-FIbu/MMC micelles) were prepared by thin film hydration method. Characterization was performed by means of ${ }^{1} \mathrm{H}-\mathrm{NMR}$, critical micelle concentration (CMC), particle size, polydispersity index (PDI), morphology, drug loading efficiency (DLE), drug loading capacity (DLC) and storage stability. The chemotherapeutic activity was comprehensively assessed in both cultured tumor cells and tumorbearing animal models. The PEG-FIbu/MMC micelles were expected to expose enhanced anticancer efficiency and low toxicity by virtue of their high accumulation in the tumor, prolonged blood circulation and excellent biocompatibility.

\section{Materials and methods}

\subsection{Materials}

MMC (mitomycin C, purity grade $=99.5 \%$ ) was obtained from Livzon Pharmaceutical Group Co., Ltd. (Guangdong, China). 2-
(4-Isobutylphenyl) propionic acid (ibuprofen, >98\%), FmocLys(Boc)-OH, $N, N^{\prime}$-dicyclohexylcarbodiimide (DCC), $4^{\prime}$-aminoacetophenone and 4-dimethylaminopyridine (DMAP) were purchased from Aladdin (Shanghai, China). Dialysis bag $\left(M_{\mathrm{w}}=\right.$ 8000 to $12000 \mathrm{Da}$ ), dichloromethane (DCM), trifluoroacetic acid (TFA) and triethylamine (TEA) were obtained from Sinopharm Chemical Reagent Co., Ltd. (Shanghai, China). Dimethyl sulfoxide (DMSO), 3-(4,5-dimethylthiazol-2-yl)-2,5-diphenyl tetrazolium bromide (MTT) were all provided by SigmaAldrich (MO, USA). Poly(ethylene glycol) methyl ether (MeOPEG-OH, MW $=2000 \mathrm{kDa}$ ) were ordered from TCI (PA, USA). Roswell Park Memorial Institute (RPMI) 1640 Medium, fetal bovine serum (FBS), penicillin-streptomycin solution, Dulbecco's phosphate-buffered saline (DPBS) and trypsin-EDTA $(0.25 \%, \mathrm{w} / \mathrm{v})$ solution were purchased from Invitrogen (NY, USA). All solvents used in this study were HPLC grade.

\subsection{Synthesis of PEG-FIbu}

$\mathrm{PEG}_{2 \mathrm{k}}$-Fmoc-Ibuprofen (PEG-FIbu) carrier was synthesized following our published method. ${ }^{18}$ The chemical structure formula of PEG-FIbu was depicted in Fig. 1. The structure of PEG-FIbu was confirmed by ${ }^{1} \mathrm{H}-\mathrm{NMR}$ in $\mathrm{CDCl}_{3}$.

\subsection{Preparation and characterization of PEG-FIbu and PEG- FIbu/MMC micelles}

PEG-FIbu/MMC micelles were prepared by means of thin-film hydration method. A weighed quantity of PEG-FIbu conjugate and MMC at different carrier/drug molar ratios (mol ratios of PEG-FIbu and MMC were $0.5: 1 ; 1: 1 ; 2.5: 1$ and $5: 1$ ) were dissolved in ethanol and mixed thoroughly. The solvent was evaporated under nitrogen flow and then trace amount of residual ethanol was removed by vacuum for $2 \mathrm{~h}$. A thin film was showed and hydrated by PBS with gentle agitating to form PEGFIbu/MMC micelles. The particle size of all PEG-FIbu and PEGFIbu/MMC micelles were measured via dynamic light scattering (DLS) using a Zetasizer (Nano ZS, Malvern Co., UK). The particle size determinations were repeated in triplicate by diluting one prepared sample into three different solutions. The CMC of PEG-FIbu was measured by employing pyrene as a fluorescence probe as mentioned previously. ${ }^{18}$ Transmission electron microscopy (TEM) was utilized to examine the morphology of PEG-FIbu and PEG-FIbu/MMC micelles. Uranyl acetate (2\%) were utilized to stain samples. 
To measure the MMC loading efficiency, unincorporated MMC was removed by centrifuging at $13000 \mathrm{rpm}$ for $10 \mathrm{~min}$ and filtrating through microporous membrane of $0.22 \mu \mathrm{m}$. The determination of MMC was conducted by a HPLC method. A Inertsil $\mathrm{C}_{18}$ column $(250 \mathrm{~mm} \times 4.6 \mathrm{~mm}, 5 \mu \mathrm{m})$ and a mobile phase consisted of water/acetonitrile $(75 / 25, \mathrm{v} / \mathrm{v})$ at the flow rate of $1.0 \mathrm{ml} \mathrm{min} \mathrm{m}^{-1}$ was used. The wavelength was performed at $365 \mathrm{~nm}$. The DLC and DLE was calculated by the formula as previously reported. ${ }^{18}$

\subsection{In vitro stability tests}

A series of PEG-FIbu/MMC micelles at different molar ratios were prepared as stated and the concentration of MMC in all micelles was maintained at $1 \mathrm{mg} \mathrm{ml}^{-1}$. Since then, the particle size and DLE of samples were estimated by DLS and HPLC at different time points. Variations of size and DLE were detected and assessment was terminated when the alteration of one of them reached significant difference.

\subsection{In vitro drug release}

The sustained release effect was investigated by a dialysis method using a dialysis bag $\left(M_{\mathrm{w}}=8000-12000 \mathrm{Da}\right)$. The PBS containing $0.5 \%(\mathrm{w} / \mathrm{v})$ Tween 80 acted as release medium were dialyzed against PEG-FIbu/MMC micelles and MMC injection at $37{ }^{\circ} \mathrm{C}$ under shaking softly. At the presupposed time points, $1 \mathrm{ml}$ of the fresh medium was replaced with $1 \mathrm{ml}$ of the release substance withdrawn from release system. The content of MMC was determined using HPLC technique as mentioned above. What's more, the release of MMC from MMC injection was determined for comparison.

\subsection{Cell culture}

4T1 and MCF-7 are metastatic mammary tumor cell lines of murine and human, respectively. A549, MGC-803 and HepG2 are corresponding to human lung, stomach and liver cancer cell lines. All these cells were cultured in a humidified atmosphere with $5 \% \mathrm{CO}_{2}$ in RPMI-1640 supplemented with $1 \%$ penicillinstreptomycin and $10 \%$ FBS at $37^{\circ} \mathrm{C}$.

\subsection{In vitro cytotoxicity study}

4T1, HepG2, MCF-7, MGC-803 or A549 cells were seeded in 96multiwell plates with RPMI-1640 medium and incubated overnight. The cells were then disposed of diverse concentrations of MMC injection, at the identical MMC concentrations of PEGFIbu/MMC micelles (PEG-FIbu to $\mathrm{MMC}=2.5: 1, \mathrm{~mol} \mathrm{~mol}^{-1}$ ) and PEG-FIbu micelles respectively. The quantity of PEG-FIbu was equivalent to those of carrier in the corresponding PEGFIbu/MMC micelles. Upon incubation for two days, $20 \mu \mathrm{L}$ of MTT in DPBS ( $5 \mathrm{mg} \mathrm{ml}^{-1}$ ) was transferred to each well, followed by incubation for $4 \mathrm{~h}$. Then medium was removed and DMSO was applied to solubilize the formazan generated by MTT. The measurements of each well were made by using a microplate reader with $490 \mathrm{~nm}$ wavelength. Untreated cells were set as a control. Cell viability was calculated as $\left[\left(\mathrm{OD}_{\text {treat }}-\mathrm{OD}_{\text {blank }}\right) /\right.$ $\left.\left(\mathrm{OD}_{\text {control }}-\mathrm{OD}_{\text {blank }}\right) \times 100 \%\right]$.

\subsection{Animals}

Female BALB/c mice ( 6 to 8 weeks) were provided by the Experimental Animal Center of Shenyang Pharmaceutical University (Shenyang, China), and raised scrupulously on the basis of the National Institute of Health's Guide lines with regard to the principles of animal care (2004). Besides, all procedures were proceeded under standard conditions and approved by the Medical Ethics Committee of Shenyang Pharmaceutical University. All animal procedures were performed in accordance with the Guidelines for Care and Use of Laboratory Animals of Shenyang Pharmaceutical University and approved by the Animal Ethics Committee of Shenyang Pharmaceutical University.

\subsection{In vivo pharmacokinetics}

The $4 \mathrm{~T} 1$ tumor-bearing $\mathrm{BALB} / \mathrm{c}$ mice were classified into two groups $(n=6)$ randomly and administrated MMC and PEGFIbu/MMC micelles via tail vein injection at equivalent doses of $1 \mathrm{mg} \mathrm{kg}{ }^{-1}$ of MMC. Blood samples were gathered in a heparinized tube at $0.083,0.166,0.25,0.5,1,1.5,2,4,6,12 \mathrm{~h}$ after intravenous administration. The plasma was acquired by centrifuging immediately at $3000 \mathrm{rpm}$ for $15 \mathrm{~min}$ of heparinized blood samples and stored at $-20{ }^{\circ} \mathrm{C}$ awaiting analysis. MMC was attained from the plasma by deproteinization using ethyl acetate and subsequently centrifuged at $12000 \mathrm{rpm}$ for $10 \mathrm{~min}$.

The plasma samples were analyzed by UPLC-MS method on an ACQUITY UPLC system equipped with Waters Quattro mass spectrometer with a triple quadrupole mass analyser (Waters Corp., Milford, MA, USA). The mobile phase consisted of water containing $0.1 \%(\mathrm{v} / \mathrm{v})$ formic acid (A) and acetonitrile (B) at the flow rate of $0.20 \mathrm{ml} \mathrm{min}{ }^{-1}$ with the addition of a Universal $\mathrm{XB}$ $\mathrm{C}_{18}$ column $(150 \mathrm{~mm} \times 2.1 \mathrm{~mm}, 1.8 \mu \mathrm{m}$; Kromat, USA). The gradient elution program for samples was listed in Table S1. $\dagger$ Positive ionisation mode and MRM scan were selected. The cone voltage and the capillary voltage were set at $42 \mathrm{~V}$ and 3.0 $\mathrm{kV}$. The desolvation temperature and the source temperature were adjusted to $350{ }^{\circ} \mathrm{C}$ and $150{ }^{\circ} \mathrm{C}$ respectively. The flow rate of the desolvation and cone gas (nitrogen) were 700 and $150 \mathrm{~L} \mathrm{~h}^{-1}$, respectively.

The pharmacokinetic parameters, including elimination half-life $\left(t_{1 / 2}\right)$, area under the curve (AUC) and clearance (CL), were calculated by fitting the blood drug pharmaceutical concentrations to a two-compartment model using WinNonlin Professional Edition Version 2.1 (Pharsight Corporation, Mountain View, California).

\subsection{Tissue distribution study}

To assess the biodistribution of MMC injection and PEG-FIbu/ MMC micelles, 4T1 syngeneic breast cancer model mice were used. When the tumor volume scratched around $400-600 \mathrm{~mm}^{3}$, mice were randomly divided into two groups $(n=6)$ and then MMC injection and PEG-FIbu/MMC micelles (PEG-FIbu to MMC $=2.5: 1, \mathrm{~mol} \mathrm{~mol}^{-1}$ ) were intravenously injected at the equimolar dose of $1 \mathrm{mg}$ MMC per kg. Mice were sacrificed at the prescribed point post-injection, major organs and tumors were 
Table 1 Characterization of drug-free and MMC-loaded $P E_{2 k}-$ Flbu micelles

\begin{tabular}{|c|c|c|c|c|c|c|}
\hline Micelles & Molar ratio & Size (nm) & PDI & DLC (\%) & DLE (\%) & Stability (h) \\
\hline $\mathrm{PEG}_{2 \mathrm{k}}-\mathrm{FIbu}$ & - & $14.6 \pm 2.8$ & 0.279 & - & - & - \\
\hline \multirow{3}{*}{$\mathrm{PEG}_{2 \mathrm{k}}-\mathrm{FIbu} / \mathrm{MMC}$} & $0.5: 1$ & $148.7 \pm 0.6$ & 0.381 & 26.2 & 65.5 & 48 \\
\hline & $2.5: 1$ & $103.2 \pm 0.9$ & 0.335 & 5.2 & 96.6 & 72 \\
\hline & $5: 1$ & $135.6 \pm 1.3$ & 0.231 & 2.6 & 98.2 & 96 \\
\hline
\end{tabular}

resected for MMC detection. MMC was recovered using ethyl acetate and centrifuged afterwards at $12000 \mathrm{rpm}$ at $4{ }^{\circ} \mathrm{C}$ for $10 \mathrm{~min}$. The MMC content of the supernatant liquid was then determined by UPLC-MS approach described above as the percentage of injected dose per $g$ of tissue.

\subsection{In vivo therapeutic study}

The 4T1 breast tumor-bearing mice were applied to explore the therapeutic efficacy of MMC formulated in PEG-FIbu micelles. Mice were inoculated with $2 \times 10^{5} 4 \mathrm{~T} 1$ cells in $100 \mu \mathrm{L}$ PBS by subcutaneous injection to establish 4T1 tumor-bearing model. Treatments were initiated when the tumor volumes scratched an approximate size of $100 \mathrm{~mm}^{3}$ and this day was designated as day 1 . On day 1 , mice were randomly sorted into four groups $(n$ $=5$ ) and received intravenous administration of PEG-FIbu/ MMC micelles (PEG-FIbu to MMC $=2.5: 1, \mathrm{~mol} \mathrm{~mol}^{-1}$ ), MMC injection at $1 \mathrm{mg}$ MMC per $\mathrm{kg}$ equivalent dose as well as blank PEG-FIbu micelles, respectively on days 1, 4, 7, 10, 13, 16, 19, 22, 25 and 28, while mice of con group received saline. Tumor volume and body weight were measured every three days and calculated according to the following formula: $\left(L \times W^{2}\right) / 2$, where $L$ is the longest and $W$ is the shortest in tumor diameters $(\mathrm{mm})$.

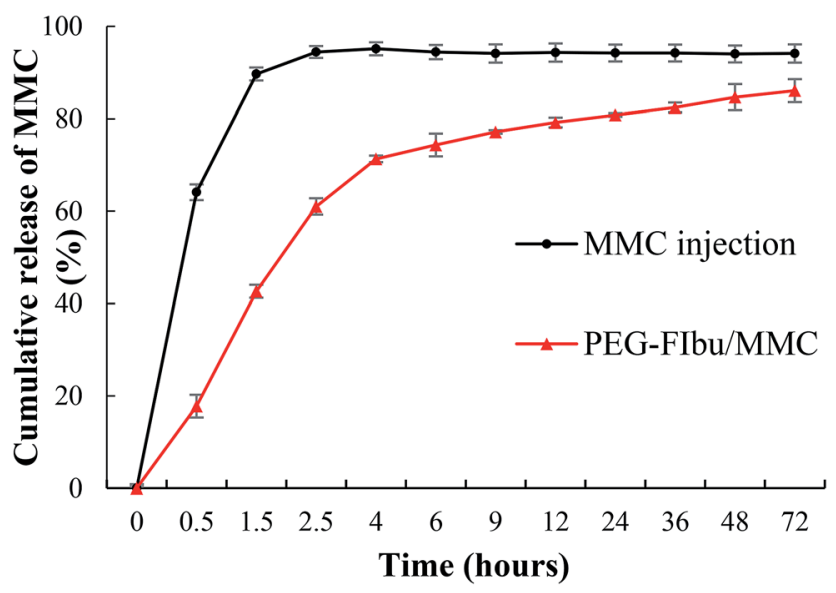

Fig. 2 Cumulative release curves of MMC and PEG-FIbu/MMC micelles.
To make a comparison in four groups, relative tumor volume (RTV) was reported at each time point (where RTV equals the tumor volume at a given time point divided by the tumor volume prior to first treatment). Mice were sacrificed when tumor reached $2000 \mathrm{~mm}^{3}$ or developed ulceration. On day 28 , the mice were executed and the tumors were resected and weighed. The serum was removed for detecting biochemical parameters. To monitor the efficacy and potential toxicity, the histopathological analysis of all tissues was carried out by means of hematoxylin and eosin (H\&E) staining. The samples were surveyed under an OLYMPUS BX51 microscope coupled with an OLYMPUS DP72 digital imaging system (Tokyo, Japan).

\subsection{Statistical analysis}

In all statistical analyses, the significance level was set to the probability of $P<0.05$. All results were presented as the mean \pm standard deviation (SD) without specification. Statistical analysis was implemented by Student's $t$-test for two groups, and one-way ANOVA for multiple groups.

\section{Results and discussion}

\subsection{Preparation and characterization of PEG-FIbu/MMC micelles}

The ${ }^{1} \mathrm{H}$-NMR spectrum of PEG-FIbu (Fig. $\mathrm{S} 1 \dagger$ ) showed peaks at $3.63,7.0-7.3,0.8$ and 2.4 ppm assigned to the protons of PEG, Fmoc motif and ibuprofen. The results confirmed the successful acquisition of PEG-FIbu polymer micelles. The CMC of PEGFIbu micelles was examined employing pyrene as a fluorescence probe and was found to be $0.202 \mu \mathrm{M}$ (Fig. S2 $\dagger$ ), which is stable upon dilution in vivo. The determination of CMC currently employed blank micelles. When $c$ (PEG-FIbu) is lower than CMC, pyrene is in polar environment in aqueous solution. When $c$ (PEG-FIbu) is higher than CMC, pyrene is in PEG-FIbu micelles, the polarity of surrounding environment is weakened, and the fluorescence peak shifted red. We thought that the presence of MMC did not change the colloidal properties of PEG-FIbu micelles. The UV-Vis spectrum of MMC had maximum absorption at 217 and $365 \mathrm{~nm}$ and PEG-FIbu had no

Table 2 EC50 values of $\mathrm{PEG}_{2 \mathrm{k}}-\mathrm{Flbu}, \mathrm{MMC}$ injection and $\mathrm{PEG}_{2 \mathrm{k}}-\mathrm{Flbu} / \mathrm{MMC}$ micelles against five tumor cell lines

\begin{tabular}{|c|c|c|c|c|c|}
\hline Cell lines & A549 & MCF-7 & MGC-803 & HepG2 & $4 \mathrm{~T} 1$ \\
\hline $\mathrm{PEG}_{2 \mathrm{k}}-\mathrm{FIbu}\left(\mu \mathrm{g} \mathrm{m}{ }^{-1}\right)$ & $32.37 \pm 2.3$ & $28.96 \pm 3.0$ & $45.09 \pm 3.3$ & $24.66 \pm 2.4$ & $34.63 \pm 1.9$ \\
\hline $\operatorname{MMC}(\mu \mathrm{g} \mathrm{ml}-1)$ & $7.55 \pm 0.9$ & $3.73 \pm 0.2$ & $8.38 \pm 1.1$ & $2.95 \pm 0.1$ & $5.12 \pm 0.1$ \\
\hline
\end{tabular}


interference at $365 \mathrm{~nm}$. So this wavelength can be used as the preferred wavelength for MMC content determination.

PEG-FIbu mixed with MMC to form a lavender uniform transparent solution after hydration. Nitrogen blowing uses the inert nature of nitrogen to prevent sample changes and protect the material from drying under conditions such as oxidation. The viscosity of ethanol is low, and a large amount of ethanol is not suitable for vacuum drying. Pre-nitrogen drying is employed to remove the main excess ethanol in the solution. The disadvantage of nitrogen blowing is that there may be residual solvent, so we take vacuum drying to remove a small amount of residual solvent. Moreover, using this method takes less time and improves the preparation efficiency. The film-forming solvent needs to have good solubility to both the drug and the
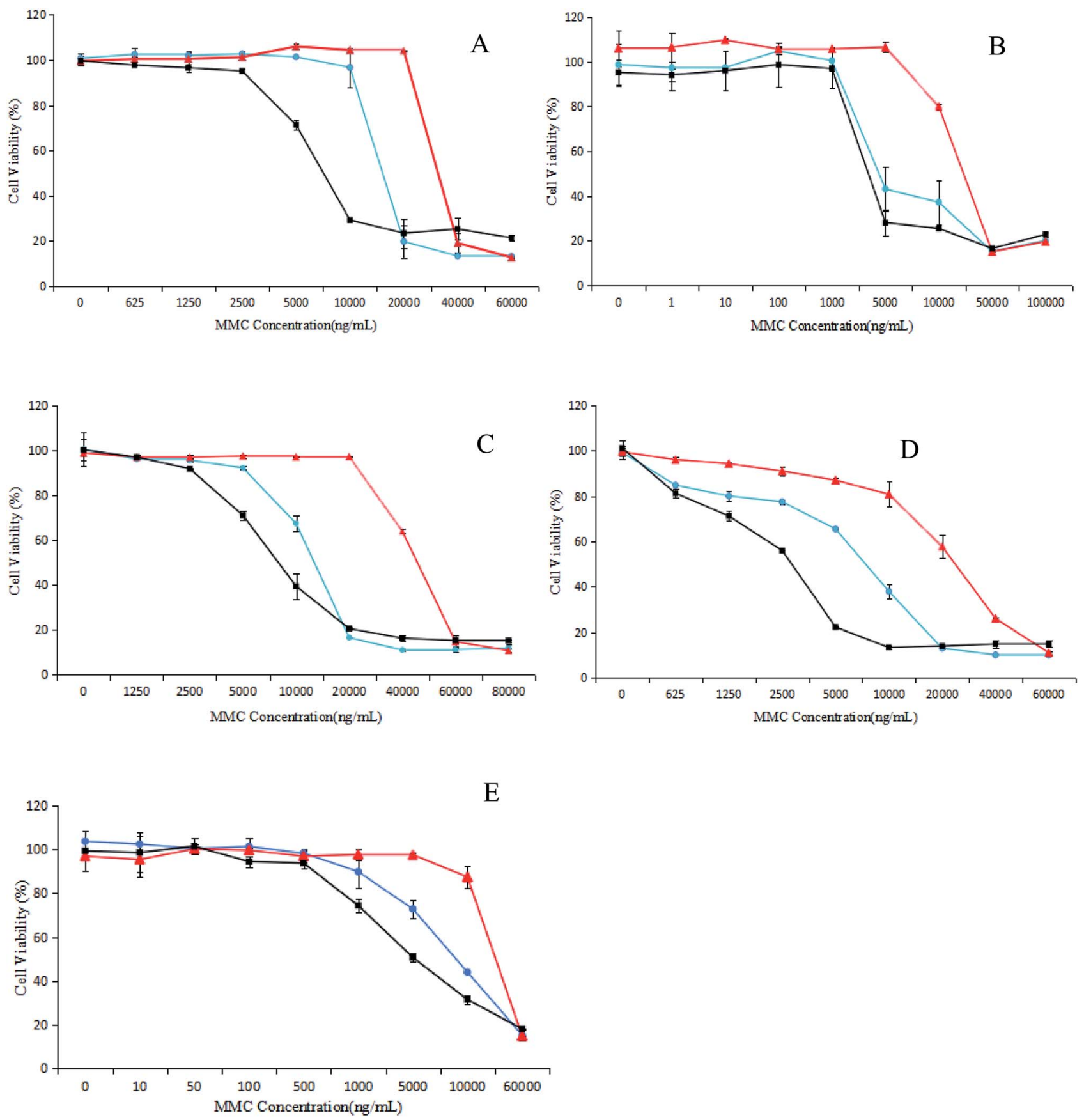

\section{PEG-FIbu/MMC - - PEG-FIbu $\because-$ MMC}

Fig. 3 Cytotoxicity of MMC, drug-free, and MMC-loaded PEG ${ }_{2 k}-$ Flbu micelles against human lung cancer cell line A549 (A), human breast cancer cell line MCF-7 (B), human gastric cancer cell line MGC-803 (C), human liver cancer cell line HepG2 (D) and murine breast cancer cell line 4T1 (E). 


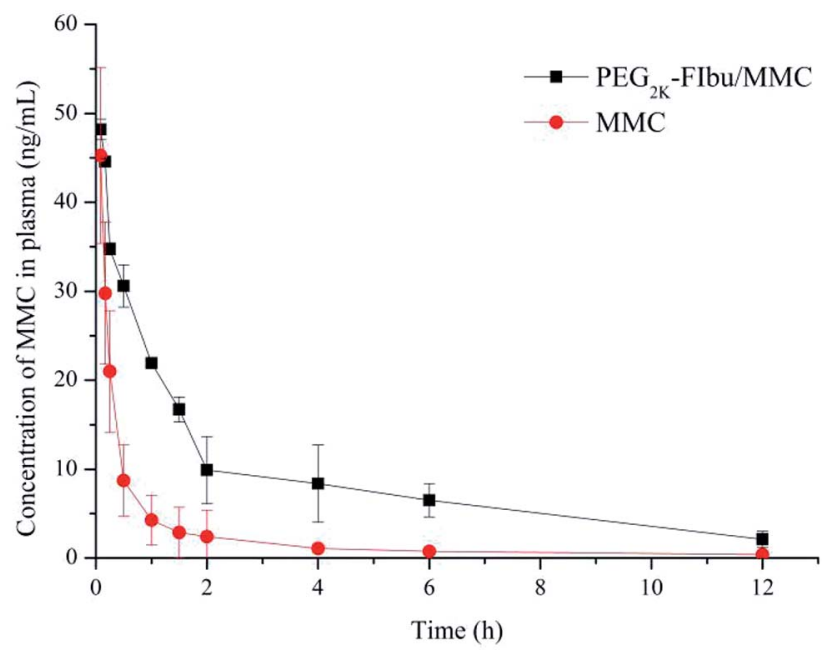

Fig. 4 Plasma concentration-time profiles of $M M C$ and $P E_{2 k}-F l b u /$ $M M C$ in mice after intravenous injection of a dose of $1 \mathrm{mg} \mathrm{kg}^{-1} M M C$.

polymer, and can be sufficiently mixed and dispersed uniformly. After the solvent was investigated, it was found that ethanol was low in toxicity, relatively volatile, and the prepared micelles had a high encapsulation efficiency, so ethanol was selected as a film-forming solvent. Since the film forming solvent needs to be completely removed, the method of drying with nitrogen before vacuum is adopted. PEG-FIbu readily formed mixed micelles with MMC in aqueous solution and loading of MMC gave rise to a significant expansion in particle size. Table 1 showed the particle size, PDI, DLC, DLE and stability at various PEG-FIbu/MMC molar ratios. The average particle size of PEG-FIbu micelles was $(14.6 \pm 2.8) \mathrm{nm}$ and PEGFIbu/MMC micelles prepared by the thin film hydration method was $(103.2 \pm 0.9) \mathrm{nm}$ when the molar ratio of PEG-FIbu/MMC was $2.5: 1$. Under this ratio, $96.6 \%$ of the MMC was loaded in PEG-FIbu micelles with a relatively high stability for $72 \mathrm{~h}$ at room temperature. As the molar ratio of PEG-FIbu to MMC increased, the DLE and stability of the PEG-FIbu/MMC micelles increased. Also, it is observed under TEM (Fig. S3 $\dagger$ ) that spherical uniform distribution of particles and sizes of the micelles were consistent with those measured by DLS (Fig. S4†). The results demonstrated the successful prepared of the PEGFIbu/MMC micelles which had narrow size, homogeneous dispersion of the particles, high drug loading efficiency and stability.

\subsection{In vitro drug release}

The profile of MMC released from PEG-FIbu/MMC micelles was showed in Fig. 2 compared to that of MMC injection. For the initial 90 min, about $90.61 \%$ of MMC was released from MMC injection, while only $52.27 \%$ of MMC been from PEG-FIbu/ MMC micelles. The release of MMC from PEG-FIbu/MMC micelles was significantly slower than that from MMC formulation. The slow kinetics of MMC release is likely due to a strong carrier/drug interaction, which may help to facilitate the MMC delivery through minimizing its premature release from PEGFIbu/MMC mixed micelles before they reach the tumor site. Overall, PEG-FIbu/MMC micelles exhibited sustained properties of MMC release compared to injection.

\subsection{In vitro cytotoxicity}

To fully investigate the cytotoxicity of PEG-FIbu/MMC micelles compared to that of MMC injection and PEG-FIbu micelles, MTT method was applied. As shown in Table 2 and Fig. 3 , the treatment of PEG-FIbu carrier alone did not reveal any significant toxicity to tumor cells until the concentration reached $20 \mu \mathrm{g} \mathrm{ml} \mathrm{m}^{-1}$. Considering lessened release rates and extent of MMC, both PEG-FIbu and PEG-FIbu/MMC micelles demonstrated less cytotoxicity than that of MMC injection in the low concentration range (0.001-20 $\mu \mathrm{g}$ $\mathrm{ml}^{-1}$ ), while no significant difference emerged among the three at high doses $\left(20 \mu \mathrm{g} \mathrm{ml}^{-1}\right.$ and above). In vitro cytotoxicity results exposed that PEG-FIbu/MMC micelles was effective in mediating intracellular delivery of MMC to tumor cells.

\subsection{Pharmacokinetics}

To evaluate the therapeutic potency of the PEG-FIbu/MMC micelles, pharmacokinetics of the PEG-FIbu/MMC micelles and MMC injection was investigated in vivo. The plasma concentration versus time profiles after intravenous administration are illustrated in Fig. 4, and pharmacokinetic parameters are shown in Table 3. The blood circulation time of PEGFIbu/MMC micelles was significantly enlarged with a longer elimination half-life $\left(t_{1 / 2}\right)$, higher area under the curve (AUC), and a substantially lower value for clearance (CL) than MMC injection. The prolonged circulation time and reduced clearance of plasma MMC in PEG-FIbu/MMC micelles group associated with its sustained release in vitro, indicating that PEGFIbu/MMC micelles changed the pharmacokinetic behavior of MMC in 4T1 tumor-bearing mice. On the whole, we concluded that PEG-FIbu micelles could be an alternative drug carrier to ameliorate the pharmacokinetic performance of MMC in vivo.

\subsection{Tissue distribution}

The tumor targeting efficiency of PEG-FIbu/MMC micelles was researched in comparison with MMC injection. As shown in Fig. 5, there was more than two-fold increase in the tumor accumulation of MMC in PEG-FIbu/MMC micelles treatment group and significant decrease in the organ regarding heart,

Table 3 Summary of pharmacokinetic parameters of $\mathrm{PEG}_{2 \mathrm{k}}-\mathrm{Flbu} / \mathrm{MMC}$ and $\mathrm{MMC}$ at a MMC equivalent dose of $1 \mathrm{mg} \mathrm{kg}^{-1}$

Pharmacokinetic

\begin{tabular}{|c|c|c|c|}
\hline parameters & $t_{1 / 2}(\mathrm{~h})$ & $\operatorname{AUC}_{0-\infty}\left(\mu \mathrm{g} \min \mathrm{L}^{-1}\right)$ & $\mathrm{CL}\left(\mathrm{L} \mathrm{h}^{-1} \mathrm{~kg}^{-1}\right)$ \\
\hline MMC & $2.46 \pm 0.95$ & $1768.68 \pm 277.86$ & $27.17 \pm 5.19$ \\
\hline $\mathrm{PEG}_{2 \mathrm{k}}-\mathrm{FIbu} / \mathrm{MMC}$ & $4.34 \pm 1.30$ & $6649.14 \pm 332.04$ & $8.34 \pm 1.04$ \\
\hline
\end{tabular}




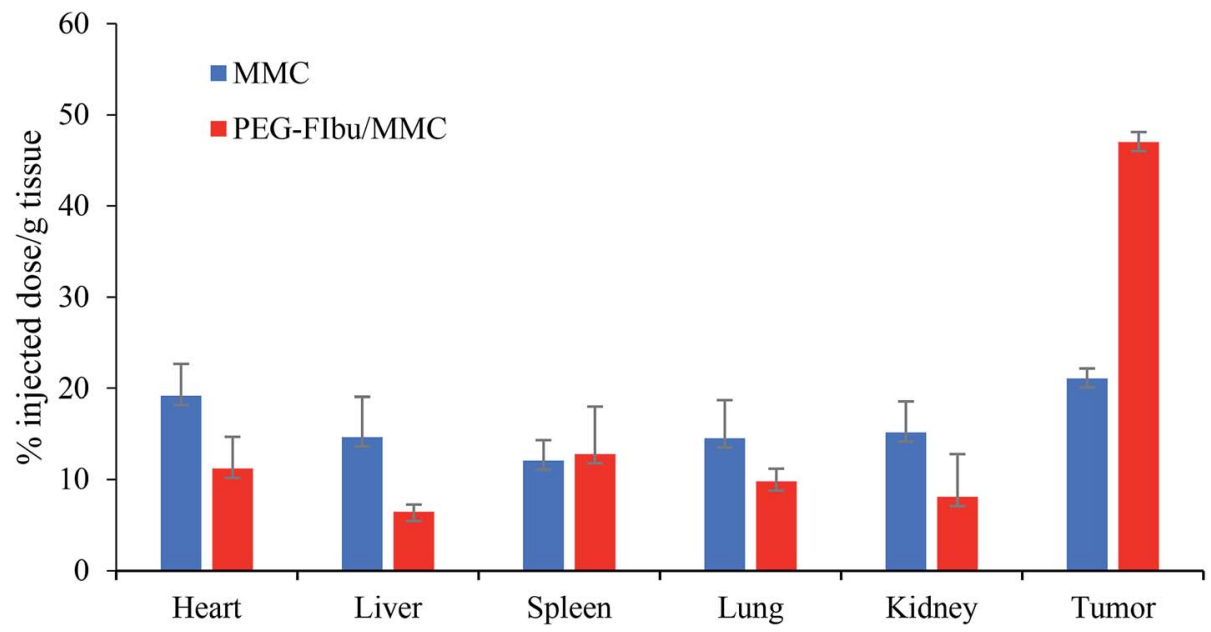

Fig. 5 Tissue distribution of MMC in mice $12 \mathrm{~h}$ following intravenous injection of a dose of $1 \mathrm{mg} \mathrm{kg}^{-1} \mathrm{MMC}_{\text {. }}$

liver, lung and spleen. The effective targeting of PEG-FIbu/MMC micelles was possibly due to the small-sized particles, stability, and long circulation. Our results acted in concert with the studies of other micellar systems with similar particle sizes. ${ }^{27-33}$ Furthermore, PEG-FIbu/MMC micelles were involved in a decreased MMC penetration in normal organs such as heart, liver, lung and spleen. The findings suggested that PEG-FIbu/ MMC micelles can increase the tumor-target capacity of MMC while reducing MMC-related toxicity.

\subsection{In vivo anticancer efficacy}

During the experiment, saline-treated mice (Con group) revealed a rapid uncontrolled increase in tumor size on account of high proliferation rate of $4 \mathrm{~T} 1$ cancer cell line, while. As observed in Fig. 6B, PEG-FIbu/MMC micelles therapy displayed maximal inhibitory effect on tumor growth among all the four treatments. In contrast, a significant inhibition of tumor growth was attained in PEG-FIbu/MMC micelles treatment group at the
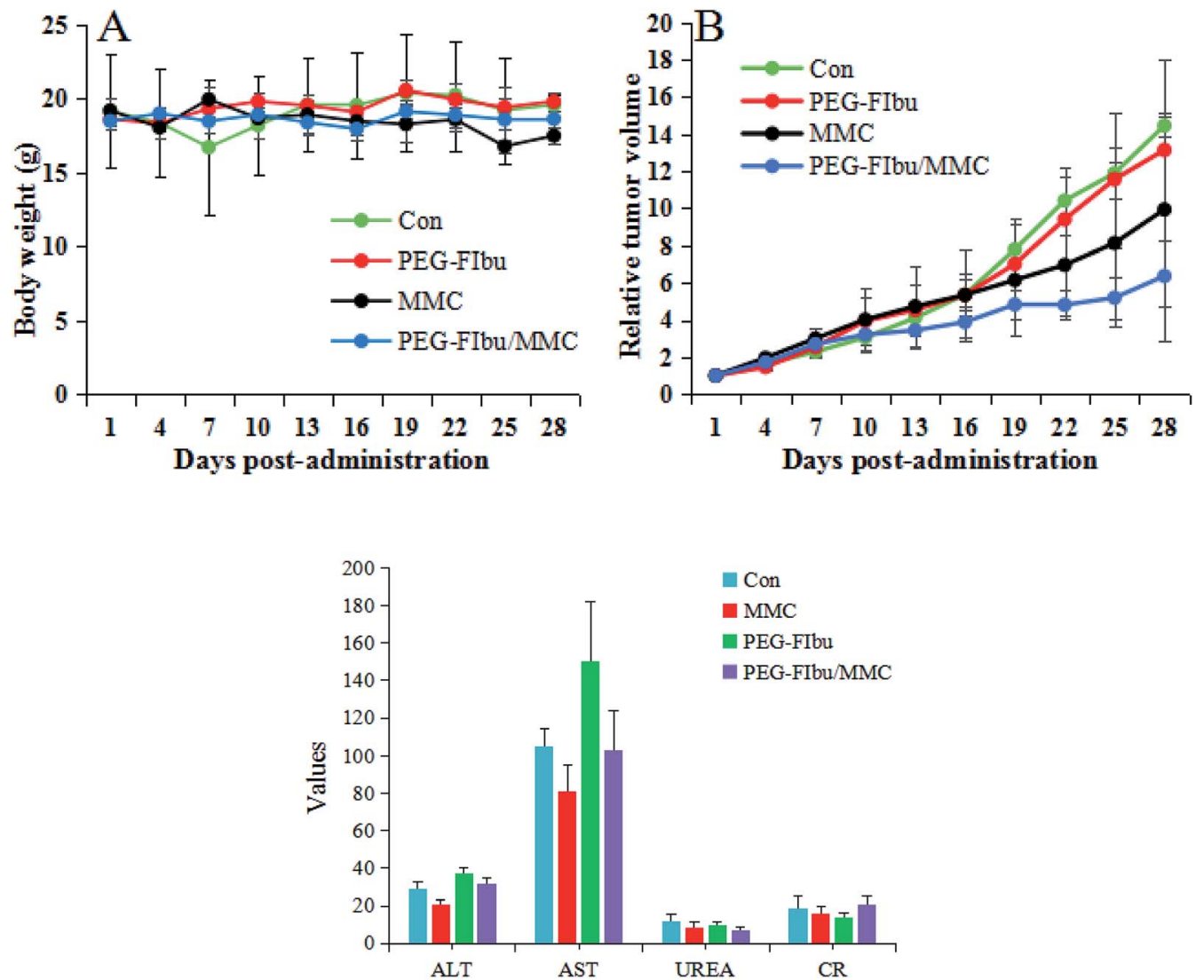

Fig. 6 Variation of body weight (A) and relative tumor volume (B) in the tumor-bearing mice with time. (C) Hematological parameters after last treatment. 


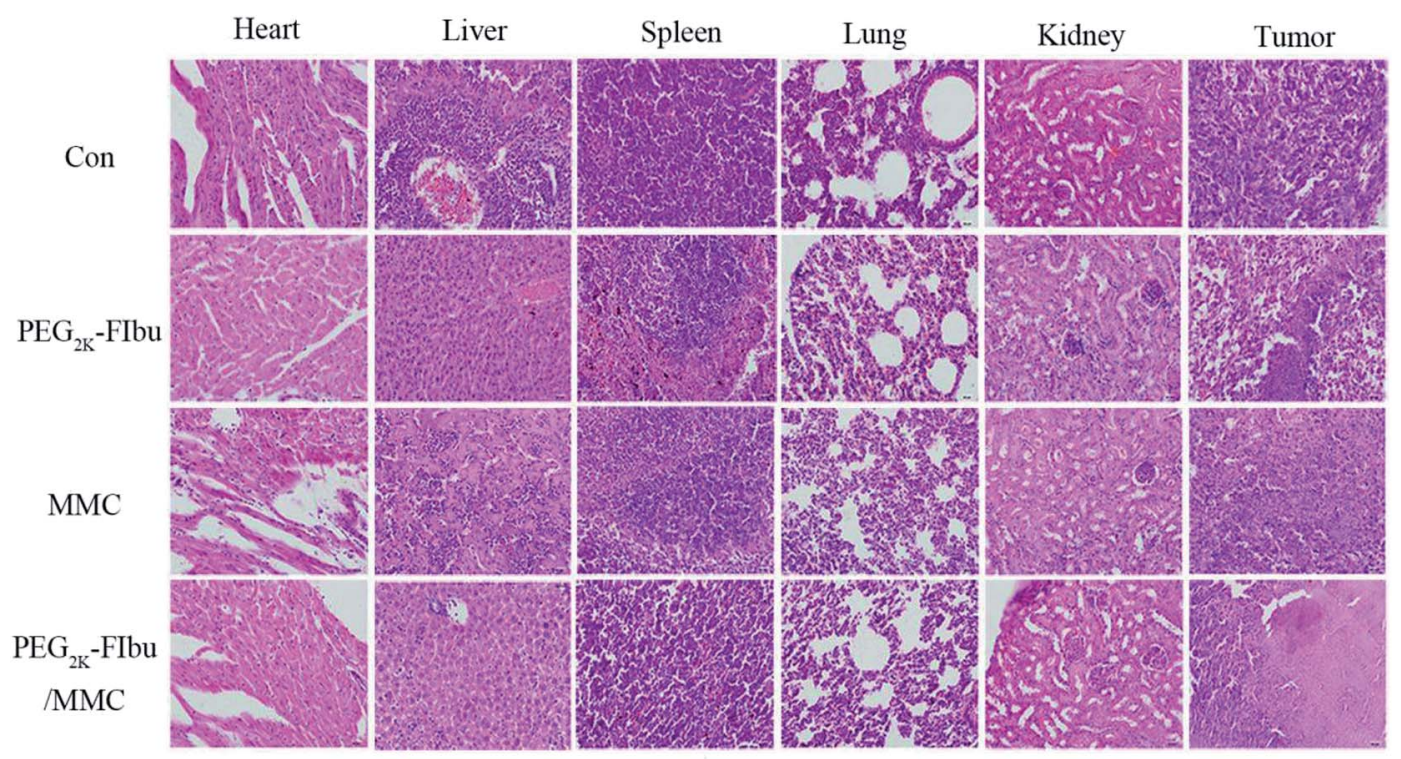

Fig. 7 Photomicrographs of histopathological sections of heart, liver, spleen, lung, kidney and tumor from the Con group, $\mathrm{PEG} \mathrm{Gk}^{-} \mathrm{Flbu}$ group, MMC group, $\mathrm{PEG}_{2 \mathrm{k}}-\mathrm{Flbu} / \mathrm{MMC}$ group.

same MMC dose of $1 \mathrm{mg} \mathrm{kg}{ }^{-1}$ as the mice treated with MMC injection, which was probably caused by the effective delivery of MMC to tumor through EPR effect. In addition, histopathologic examination of tumors from $4 \mathrm{~T} 1$ tumor-bearing mice was carried out to further explore the therapeutic efficacy of PEGFIbu/MMC micelles. Micrographs of pathological tumor tissue sections were illustrated in Fig. 7. Con group appeared visible dense tumor cell sheets with potent tumor cell division due to high proliferation rate. PEG-FIbu micelles treatment showed a certain extent of antitumor activity, as visible degeneration of tumor cells could be observed. Tumors in MMC group appeared morphological changes with shrunk nucleolus and reduced tumor cell density, indicating remarkable cell death after treatment with MMC injection. As compared to other three therapies, not even one live tumor cell was noticed on treatment with PEG-FIbu/MMC micelles.

The toxicity of all therapies was assessed by tracking variety on body weight, serum biochemical parameters and the H\&E stained images. As indicated in Fig. 6A, a decrease of body weight was discovered in MMC injection therapy group while no significant changes in body weight were found in other therapies, which was likely attributed to the side effects of MMC distributed to normal organs. Hematological parameters (Fig. 6C) suggested that MMC administration significantly caused an increase in AST levels, indicating that liver injury in mice, and PEG-FIbu had a tendency to improve the function of liver and kidney.

Moreover, the toxicity was validated by H\&E stained tissue sections of major organs. MMC group exhibited myocardial rupture, renal tubules damage, large range of cell necrosis in liver tissue compared to the Con group, indicating that MMC has cardiotoxicity, hepatotoxicity and nephrotoxicity. In contrast, PEG-FIbu/MMC group suggested that the necrotic cells were significantly reduced in liver tissue and no obvious abnormalities or damage were found in the heart, spleen, lung and kidney. It was noted that PEG-FIbu had a certain protective effect on the toxicity of MMC and significantly reduced the side effects of MMC.

Thus, the in vivo anticancer efficacy represented that increased therapeutic capacity could be achieved with minimal toxicity in PEG-FIbu/MMC micellar system and provided more convincing evidence for the anticancer potential of the PEG-FIbu micelles.

\section{Conclusions}

In summary, PEG-FIbu/MMC micelles were successfully prepared by thin film hydration technique and evaluated in vitro and in vivo. This research clearly showed that PEG-FIbu/MMC micelles not only presented reasonable particle size and low CMC value, excellent stability, high drug loading efficiency, sustained release, but also demonstrated prolonged blood circulation, significantly higher tumor uptake and therapeutic activity and lower toxicity than MMC injection. Therefore, the PEG-FIbu/MMC micelles can be regarded as effective nano delivery system and have broad application potential for targeted combination cancer chemotherapy. Considering that inflammation is constantly associated to cancer and the metastasis of the tumor itself can induce immune response, immunological studies such as white blood cell counts will continue to be investigated in the future.

\section{Conflicts of interest}

The authors report no conflicts of interest. The authors alone are responsible for the content and writing of this article.

\section{Acknowledgements}

This work was supported by the Natural Science Foundation of Liaoning Province of China (Grant no. 20170520254). 


\section{References}

1 Z. C. Soe, B. K. Poudel, H. T. Nguyen, R. K. Thapa, W. Ou, M. Gautam, et al., Folate-targeted nanostructured chitosan/ chondroitin sulfate complex carriers for enhanced delivery of bortezomib to colorectal cancer cells, Asian J. Pharm. Sci., 2019, 14(1), 40-51.

2 Y. Li, J. Lin, H. Wu, M. Jia, C. Yuan, Y. Chang, et al., Novel methotrexate prodrug-targeted drug delivery system based on PEG-lipid-PLA hybrid nanoparticles for enhanced anticancer efficacy and reduced toxicity of mitomycin C, $J$. Mater. Chem. B, 2014, 2, 6534-6548.

3 X. Wei, Y. Patil, P. Ohana, Y. Amitay, H. Shmeeda, A. Gabizon, et al., Characterization of pegylated liposomal mitomycin C lipid-based prodrug (promitil) by high sensitivity differential scanning calorimetry and cryogenic transmission electron microscopy, Mol. Pharmaceutics, 2017, 14(12), 4339-4345.

4 M. Kotmakchiev, G. Kantarcı, V. Cetintas, et al., Cytotoxicity of a novel oil/water microemulsion system loaded with mitomycin C in in vitro lung cancer models, Drug Dev. Res., 2012, 73(4), 185-195.

5 S. Zalipsky, M. Saad, R. Kiwen, E. Ber, N. Yu and T. Minko, Antitumor activity of new liposomal prodrug of mitomycin C in multidrug resistant solid tumor: insights of the mechanism of action, J. Drug Targeting, 2007, 15(7-8), 518530.

6 R. Y. Cheung, Y. Ying, A. M. Rauth, N. Marcon and W. X. Yu, Biodegradable dextran-based microspheres for delivery of anticancer drug mitomycin C, Biomaterials, 2005, 26, 53755385.

7 A. Gabizon, Y. Amitay, D. Tzemach, J. Gorin, H. Shmeeda and S. Zalipsky, Therapeutic efficacy of a lipid-based prodrug of mitomycin $\mathrm{C}$ in pegylated liposomes: studies with human gastro-entero-pancreatic ectopic tumor models, J. Controlled Release, 2012, 160, 245-253.

8 Z. Hou, Y. Li, Y. Huang, C. Zhou, J. Lin, Y. Wang, et al., Phytosomes loaded with mitomycin C-soybean phosphatidylcholine complex developed for drug delivery, Mol. Pharmaceutics, 2013, 10, 90-101.

9 J. Lin, Y. Li, H. Wu, X. Yang, Y. Li, S. Ye, et al., Tumortargeted co-delivery of mitomycin $\mathrm{C}$ and 10hydroxycamptothecin via micellar nanocarriers for enhanced anticancer efficacy, RSC Adv., 2015, 5, 2302223033.

10 Y. Li, H. Wu, X. Yang, M. Jia, Y. Li, Y. Huang, et al., Mitomycin C-soybean phosphatidylcholine complex-loaded self-assembled PEG-Lipid-PLA hybrid nanoparticles for targeted drug delivery and dual-controlled drug release, Mol. Pharmaceutics, 2014, 11(8), 2915-2927.

11 A. A. Gabizon, D. Tzemach, A. T. Horowitz, H. Shmeeda, J. Yeh and S. Zalipsky, Reduced toxicity and superior therapeutic activity of a mitomycin $\mathrm{C}$ lipid-based prodrug incorporated in pegylated liposomes, Clin. Cancer Res., 2006, 12, 1913-1920.
12 Z. Hou, H. Wei, Q. Wang, Q. Sun, C. Zhou, C. Zhan, et al., New method to prepare mitomycin $\mathrm{C}$ loaded PLAnanoparticles with high drug entrapment efficiency, Nanoscale Res. Lett., 2009, 4, 732-737.

13 H. Maeda, J. Wu, T. Sawa, Y. Matsumura and K. Hori, Tumor vascular permeability and the EPR effect in macromolecular therapeutics: a review, J. Controlled Release, 2000, 65, 271284.

$14 \mathrm{H}$. Maeda, The enhanced permeability and retention (EPR) effect in tumor vasculature: the key role of tumor-selective macromolecular drug targeting, Adv. Enzyme Regul., 2001, 41, 189-207.

15 X. Zhang, Y. Huang and S. Li, Nanomicellar carriers for targeted delivery of anticancer agents, Ther. Delivery, 2014, 5(1), 53-68.

16 R. Thipparaboina, R. B. Chavan, D. Kumar, S. Modugula and N. R. Shastri, Micellar carriers for the delivery of multiple therapeutic agents, Colloids Surf., B, 2015, 135, 291-308.

17 K. M. Huh, S. C. Lee, Y. W. Cho, J. Lee, J. H. Jeong and K. Park, Hydrotropic polymer micelle system for delivery of paclitaxel, J. Controlled Release, 2005, 101, 59-68.

18 M. Zhao, Y. Huang, Y. Chen, J. Xu, S. Li and X. Guo, PEGFmoc-Ibuprofen Conjugate as a Dual Functional Nanomicellar Carrier for Paclitaxel, Bioconjugate Chem., 2016, 27(9), 2198-2205.

19 X. Guo, Z. Zhao, D. Chen, M. Qiao, F. Wan, D. Cun, et al., Codelivery of resveratrol and docetaxel via polymeric micelles to improve the treatment of drug-resistant tumors, Asian J. Pharm. Sci., 2019, 14(1), 78-85.

20 P. Zhang, Y. Huang, H. Liu, R. T. Marquez, J. Lu, W. Zhao, et al., A PEG-Fmoc conjugate as a nanocarrier for paclitaxel, Biomaterials, 2014, 35, 7146-7156.

21 P. Zhang, J. Lu, Y. Huang, W. Zhao, Y. Zhang, X. Zhang, et al., Design and evaluation of a PEGylated lipopeptide equipped with drug-interactive motifs as an improved drug carrier, AAPS J., 2014, 16, 114-124.

22 X. Gao, Y. Huang, A. M. Makhov, M. Epperly, J. Lu, S. Grab, et al., Nanoassembly of surfactants with interfacial druginteractive motifs as tailor-designed drug carriers, Mol. Pharmaceutics, 2013, 10, 187-198.

23 A. Hegmans, Y. Qu, L. R. Kelland, J. D. Roberts and N. Farrell, Novel Approaches to Polynuclear Platinum ProDrugs. Selective Release of Cytotoxic Platinum-Spermidine Species through Hydrolytic Cleavage of Carbamates, Inorg. Chem., 2001, 40(24), 6108-6114.

24 P. Zhang, J. Li, M. Ghazwani, W. Zhao, Y. Huang, X. Zhang, et al., Effective co-delivery of doxorubicin and dasatinib using a PEG-Fmoc nanocarrier for combination cancer chemotherapy, Biomaterials, 2015, 67, 104-114.

25 P. Bonelli, F. M. Tuccillo, R. Calemma, F. Pezzetti, A. Borrelli, R. Martinelli, et al., Changes in the gene expression profile of gastric cancer cells in response to ibuprofen: a gene pathway analysis, Pharmacogenomics J., 2011, 11, 412-428.

26 J. Andrews, D. Djakiew, S. Krygier and P. Andrews, Superior effectiveness of ibuprofen compared with other NSAIDs for reducing the survival of human prostate cancer cells, Cancer Chemother. Pharmacol., 2002, 50(4), 277-284. 
27 N. Zheng, W. Dai, W. Du, H. Zhang, L. Lei, H. Zhang, et al., A novel lanreotide-encoded micelle system targets paclitaxel to the tumors with overexpression of somatostatin receptors, Mol. Pharmaceutics, 2012, 9, 1175-1188.

28 X. Zhang, K. Liu, Y. Huang, J. Xu, J. Li, X. Ma, et al., Reduction-sensitive dual functional nanomicelles for improved delivery of paclitaxel, Bioconjugate Chem., 2014, 25, 1689-1696.

29 X. Zhang, Y. Huang, W. Zhao, H. Liu, R. Marquez, J. Lu, et al., Targeted delivery of anticancer agents via a dual function nanocarrier with an interfacial drug-interactive motif, Biomacromolecules, 2014, 15, 4326-4335.

30 J. Y. Kim, S. Kim, R. Pinal and K. Park, Hydrotropic polymer micelles as versatile vehicles for delivery of poorly watersoluble drugs, J. Controlled Release, 2011, 152, 13-20.
31 G. Saravanakumar, K. H. Min, D. S. Min, A. Y. Kim, C. M. Lee, Y. W. Cho, et al., Hydrotropic oligomer-conjugated glycol chitosan as a carrier of paclitaxel: synthesis, characterization, and in vivo biodistribution, J. Controlled Release, 2009, 140, 210-217.

32 P. Zhang, Y. Huang, Y. T. Kwon and S. Li, PEGylated FmocAmino Acid Conjugates as Effective Nanocarriers for Improved Drug Delivery, Mol. Pharmaceutics, 2015, 12(5), 1680-1690.

33 H. Zhang, K. Wang, K. Na, D. Li, Z. Li, D. Zhao, et al., Striking a balance between carbonate/carbamate linkage bond and reduction-sensitive disulfide bond-bearing linker for tailored controlled release: in situ covalent-albuminbinding gemcitabine prodrugs promote bioavailability and tumor accumulation, J. Med. Chem., 2018, 61(11), 4904-4917. 